\title{
Defining Neglected Disease
}

\begin{abstract}
$\underline{\text { Abstract }}$
In this paper I seek to say what it is for something to count as a neglected disease. I argue that neglect should be defined in terms of efforts at prevention, mitigation and cure, and not solely in terms of research dollars per DALY. I further argue that the trend towards multifactorialism and risk factor thinking in modern epidemiology has lent credibility to the erroneous view that the primary problem with neglected diseases is a lack of research. A more restrictive contrastive model of disease is endorsed as better suited to the definition of neglected disease.
\end{abstract}

\section{Introduction}

A number of diseases are listed as neglected by organizations such as the World Health Organization (WHO) (World Health Organisation 2010a). But inclusion on a list means little, unless there are identifiable criteria for inclusion on that list. So it is unfortunate that there is no precise and broadly accepted definition of neglected diseases (Burri 2004). The purpose of this paper is to attempt a principled and reasonably clear statement of the conditions that a disease must satisfy in order to be counted as a neglected disease. In principle we are free to define the words as we like, but I count a good definition of neglected disease as one that will help us with associated practical goals: saying what is wrong about the fact these diseases are neglected, and working out what to do about them.

In order to say what a neglected disease is, we must say what neglect is in this context. In Section 2 I argue that neglect is not best defined in terms of money spent on research and development (R\&D). It is possible to reduce the disease burden of many of these diseases without further R\&D investment, and R\&D investment is not necessarily the best way to reduce the burden of these diseases. I suggest that R\&D 
investment is necessary for identifying profitable ways to reduce the disease burden; but defining neglected diseases in terms of neglected opportunities for profit does not seem to capture what is wrong about that neglect. Instead I propose a broader definition of neglect, including the differential availability of existing curative and preventive measures between populations, as well as differences in the levels of effort (e.g. R\&D investment) devoted to identifying such methods where they do not already exist.

The other aspect of defining neglected disease is saying what a disease is. This is a philosophically more onerous task. In Section 3 I introduce a trend in modern epidemiological thinking about disease, multifactorialism. In Section 4 I argue that the way multifactorial thinking about disease has developed reflects the challenges facing epidemiologists in countries which have passed through the epidemiological transition (where chronic and non-communicable diseases replace infectious diseases as the main cause of mortality). But neglected diseases typically occur in countries that have not passed through the epidemiological transition. Unsurprisingly, I conclude that multifactorialism as it stands is not much help for defining neglected diseases. In Section 5 I endorse a more precise development of the multifactorial model, the contrastive model of disease, and argue that it provides a more apt definition of neglected diseases. Section 6 concludes. ${ }^{1}$

\section{$\underline{\text { 2. Neglect }}$}

Defining neglected disease requires saying what neglect amounts to in this context. Although various organizations list the diseases they take to be neglected, there is no clear and generally accepted position on what merits inclusion on this list, which may explain why different institutions produce slightly different lists (Burri 2004). At time of writing, the World Health Organization lists the following health conditions as

\footnotetext{
${ }^{1}$ The neglected diseases are sometimes called the neglected tropical diseases, but I do not propose to define "tropical". Moreover I take it that what matters about these diseases is that they are neglected, not that they are tropical. Neglected arctic diseases would be no less objectionable.
} 
covered by its Neglected Tropical Disease Department (World Health Organisation 2010b):

- Buruli Ulcer

- Chagas disease(American trypanosomiasis)

- Dengue/dengue haemorrhagic fever

- Dracunculiasis (guinea-worm disease)

- Fascioliasis

- Human African trypanosomiasis

- Leishmaniasis

- Leprosy

- Lymphatic filariasis

- Onchocerciasis

- Schistosomiasis

- Soil transmitted helminthiasis

- Snakebite

- Trachoma

- Yaws

It is also quite common for research papers to take a list-based approach to definition. ${ }^{2}$ A list can specify the set of diseases to which the term applies, but it does not tell us in virtue of what shared features these diseases are grouped together. Therefore it cannot help us decide when to add or remove items, or how to go about making the list shorter. For this, we need a principled definition.

Although it does not define neglect, the WHO does offer an explanation as to why these diseases are said to be neglected:

They are named neglected because these diseases persist exclusively in the poorest and the most marginalized communities, and have been largely eliminated elsewhere and thus are often forgotten... Most can be prevented or eliminated. (World Health Organisation 2010b)

\footnotetext{
${ }^{2}$ For example, this is the approach of (Trouiller et al. 2002)
} 
Following this lead, it is clear that neglected diseases must afflict a reasonable number of people, reasonably severely. They must impose a significant health burden on some population. ${ }^{3}$ Diseases that are extremely rare may be neglected in the sense that little attention is paid to them, but what makes the neglected diseases as they are usually listed so striking is that so little attention is paid to them despite the fact that they are common in some populations. Likewise, they are without exception unpleasant for their sufferers, and often debilitating, with attendant economic and social consequences. A very mild health condition - for example, inconsequential blisters due to wearing ill-fitting shoes - would not merit inclusion on a neglected disease list.

To count as neglected, in this context, a disease must impose a significant health burden on some population. What else? Again, following the WHO's lead, one obvious way to define neglect would be in terms of the ready availability of treatments in some areas or to some people, but not others. However, a definition of neglect in terms of availability of treatment alone would be too hasty. While some neglected diseases are easy to treat with the right medical resources, for others treatment is currently difficult anywhere. For example, the drugs used for treating Chagas disease are "ineffective and toxic" (Trouiller et al. 2002, 2193). Again, the only cure for guinea worm is painful and rudimentary, waiting for parts of the worm to emerge from the lower limbs and then pulling it out a few centimetres at a time over a period of weeks (The Carter Center Guinea Worm Disease Eradication Program 2010).

This suggests that preventability must be included alongside treatability as a key feature of neglected diseases. Even if some of these diseases are difficult to treat, many are preventable by public health measures that are technologically feasible. The distribution of the disease burden can illustrate this fact, where it is attributable to the differential implementation of such measures.

\footnotetext{
${ }^{3}$ Assuming that the measure of health burden balances number of people affected and severity of effect in some reasonable way. Measuring health burdens is not the topic of this paper.
} 
As well as being prevented and cured, diseases can be mitigated, or managed. ${ }^{4}$ It may be that neither prevention nor cure for a disease exists, yet the prognosis is considerably better for some sufferers than others, due to the differential availability of methods for managing the disease. So we must also include mitigation in our definition of neglect.

However, even if we mention treatment, prevention and mitigation, our definition of neglect will not be complete. There may exist disease distributions which it is not currently feasible to prevent, cure or mitigate, and yet for which strenuous efforts to identify means of prevention, cure and mitigation are not made. In particular, it may be that a disease would be the subject of a great deal of biomedical research if it afflicted people with more money; but because it afflicts people who are very poor, very little research effort is directed towards it. This could arise, for example, if the disease was geographically limited due to climate or terrain, and the people who inhabited the affected regions were very poor.

It is difficult to identify any actual neglected disease with these features. The neglected diseases could, by and large, be eliminated by public health programs without the need for new biomedical technology. This is most obvious for those diseases which afflict only the poor even within the areas where they are prevalent, for example those which are transmitted by dirty drinking water. If some people can avoid a disease in an area where it is prevalent, that strongly suggests that prevention must be possible, by extending the means by which those who avoid the disease do so to those who get the disease. But it also holds for those neglected diseases which afflict rich and poor alike in the areas where they are prevalent. Dengue fever is such a disease (Reiss and Kitcher 2009, 266). It is transmitted by mosquito bite, and is susceptible to public health programs aimed at controlling this vector, despite the current lack of a vaccine (World Health Organisation 2010c).

Nevertheless, there certainly are neglected diseases for which further research would considerably aid public health efforts. Dengue fever is one of them. Others include Chagas disease (already mentioned), human African trypanosomiasis, and - if it is

\footnotetext{
${ }^{4}$ I am grateful to an anonymous referee for this point.
} 
counted as a neglected disease - malaria (e.g. Trouiller et al. 2002). ${ }^{5}$ In particular, there may be scope for improving existing treatments or overcoming drug-resistance (Trouiller et al. 2002).

Accordingly I propose to define neglect like this:

NEGLECT A disease $D$ is neglected to the extent that it poses a significant health burden on some reasonably large population, and preventive, curative or mitigatory measures are either widespread among other populations, or would be much more actively sought if $D$ imposed a similar burden on other populations.

This appears to capture what is morally objectionable about the existence of neglected diseases. ${ }^{6}$ It is not merely that some people suffer from these diseases while others do not; that is true of every disease. Rather, it is that some suffer while others do not because only some have the means to prevent or cure the disease, or to bring about the improvement and discovery of methods of prevention or cure.

The principal difficulty that this definition of NEGLECT faces is that it is at odds with another widely-adopted approach to neglect. That approach sees neglect as a function of investment in research and development. For example, Julian Reiss and Philip Kitcher compare R\&D funding per disability-adjusted life-year (DALY) for a number of diseases. On this basis they argue that biomedical research is not "well-

\footnotetext{
${ }^{5}$ Malaria is a good example of a disease whose neglect is contested: it does not feature on the WHO's list but other authorities, such as Trouiller et al. (2002), count it as neglected. A definition of neglect would help decide such differences, if that matters.

${ }^{6}$ Would this definition be more robust if it identified the actor? I am grateful to an anonymous referee for raising this question. The answer is No, because neglect is a failure to act in a particular way, so there is no act, and hence no actor. We could identify the "agent" of neglect as the person(s) whose failure to act counts as neglect, presumably because of a duty to act. But I think it is reasonable, in some circumstances, to say that neglect occurs without saying whose derogation of duty counts as neglect. You can say that a child has been neglected without knowing whose duty it was to look after the child, because there is a presumption that it is always somebody's duty to look after a child. I suggest that a similar presumption operates in the case of unpleasant diseases that are widespread despite being preventable or curable.
} 
ordered", meaning that its inquiries are not directed in ways that promote the common good (Reiss and Kitcher 2009; well-ordered science is discussed at length in Kitcher 2001).

While Reiss and Kitcher may well be right that biomedical research is not wellordered, it is important not to confuse scientific neglect with neglect more generally. There are other kinds of neglect. Moreover, as we saw, for most of the neglected diseases, the most important kind of neglect for the goal of reducing the disease burden is not a lack of research, because for many neglected diseases, it is already technologically feasible to reduce the disease burden by either prevention or cure.

The point is not merely academic. Confusing neglect simpliciter with neglect by biomedical science has heavily influenced some of the proposed courses of action for reducing the neglected disease burden. Many such schemes focus on encouraging pharmaceutical companies to invest in $\mathrm{R} \& \mathrm{D}$ in respect of neglected diseases. For example, Thomas Pogge proposes that financial rewards for the development of new drugs would be in proportion to the impact of those drugs on global disease burden (Pogge 2005). Reiss and Kitcher also maintain that the solution lies in adjusting the incentive structure for $R \& D$ investment, though they propose to achieve it by slightly different means. ${ }^{7}$

But as we have already seen, the majority of neglected diseases are readily treatable or preventable through means that are already available. For example, yaws is an infectious disease affecting bones and soft tissue, passed on by contact with an infected person. It is treatable using penicillin, and has been eradicated in many parts of the world. It is caused by spirochete bacterium and humans are the only reservoir of infection. In the 1950s, public health programs dramatically reduced the number of cases globally, and complete global eradication appears to be a possibility. India recently eradicated the disease (no new cases since 2004).

\footnotetext{
${ }^{7}$ Namely, tweaking the intellectual property regime to make the American drug market less profitable, but diverting part of the difference between old- and newregime prices (presumably by a tax on generic drugs?) to setting up a global organization to co-ordinate biomedical research (Reiss and Kitcher 2009, 277-80).
} 
What would be the use of creating incentives for pharmaceutical companies to invest in more R\&D in respect of yaws? The disease does not persist because we lack a cure. So there is no point trying to find one: partly because we already have one, and partly because whatever prevents the effective use of that cure is likely to prevent the use of any similar cure.

It might be replied that the cure we have is insufficient. The penicillin in question is benzathine penicillin, most usually pre-filled single dose syringes marketed as Bicillin by King Pharmaceutical. Bicillin is a suspension requiring refrigeration, which is challenging in a developing world setting. Moreover it requires intramuscular injection, which in turn requires significant medical training, and this may be lacking in the settings where yaws is found (Fegan et al. 2010, 68-9). So perhaps there is a place for R\&D into new drugs for yaws after all - to achieve a more portable, easilyadministered cure. ${ }^{8}$

It may be true that $R \& D$ into yaws could be $u$ seful. The question I am asking, however, is whether it is useful enough to be a priority: whether it is more useful than any other expenditure. Given that the existing cure has in fact been used so successfully in many parts of the world, this seems unlikely. The point is that R\&D is not necessarily the most effective strategy for reducing the burden of a neglected disease. Yaws illustrates the point because it has already been successfully eradicated in countries such as India, suggesting that adequate means to control it already exist.

What R\&D regarding yaws might do, however, is help identify profitable ways to reduce the disease burden. Though they are nobly motivated, proposals such as Pogge's, which focus on incentivizing $R \& D$, are self-defeating in this respect. It is understandable why such proposals should focus on R\&D: as Reiss and Kitcher illustrate, there is a stark contrast between the vast profits made by pharmaceutical companies and their lack of investment in neglected disease (Reiss and Kitcher 2009, 266-71). But investing in neglected disease R\&D would, necessarily, amount to

\footnotetext{
${ }^{8}$ I am grateful to an anonymous referee for pressing this point and educating me about penicillin.
} 
investing in profitable ways to reduce their burden. ${ }^{9}$ Even if one holds that the only way to achieve a goal is to render it profitable for somebody to achieve it, incentives to develop a new drug represent a very inefficient form of incentive in the case of yaws, given the costs of drug development.

Moreover, one need not subscribe to the view that the only way to get something done is to make it directly commercially viable. One might insist that health is a precondition of economic activity, and thus that public health programs can and ought to be successfully implemented (on economic grounds as well as justice-based ones) even if their implementation is not directly commercially profitable. If so, then presumably the oft-cited $\$ 800$ million (DiMasi, Hansen, and Grabowski 2003) spent on developing a new drug for yaws would be more efficiently spent on distributing penicillin. It cannot be assumed that commercial vigour would enable some new drug to overcome whatever barriers currently exist to the distribution of penicillin.

Yaws is not unusual among neglected diseases. Many are preventable or treatable by means that are within our grasp. It is true that little money is invested in R\&D in respect of the neglected diseases; but it does not follow that this is unjust in every case, nor that in every case this is an instance of biomedical research failing to be well-ordered. In the case of yaws, lack of current R\&D investment is a consequence of the striking success of previous $\mathrm{R} \& \mathrm{D}$, which produced penicillin. Lack of research is one kind of neglect, but only one kind; and for many of the neglected diseases it is not the most important kind. For this reason, neglect should be defined in terms of availability of treatment or preventive measures, as well as lack of research, along the lines I have indicated above.

\section{Multifactorialism and risk factor thinking}

The other part of the task of defining neglected diseases is defining disease: that is, saying what it takes for something to count as a disease. In this section I explain how modern epidemiology thinks about disease, in contrast to older approaches. In Section

${ }^{9}$ Because otherwise it would not be an investment, in the commercial sense, but some kind of charitable activity. 
4 I will explore the consequences for the science and politics of neglected diseases, before endorsing a definition of disease in Section 5.

Epidemiology textbooks generally trace the roots of epidemiology to the nineteenth century, and a number even refer (not altogether plausibly) to certain remarks of Hippocrates'. However, modern epidemiology, as I shall use the term, developed in Europe and America in the second half of the twentieth century, in response to the increased burden on population health from chronic non-communicable diseases, as opposed to infectious diseases (Saracci 2010, 9). ${ }^{10}$ Paradigmatic studies include the Doll-Hill work on the relationship between smoking and various cancers, especially of the lung, ${ }^{11}$ and the Framingham Heart Study, ongoing since 1948 (Framingham Heart Study 2010). In both cases, technologically simple methods - what could be simpler than counting? - proved highly successful at identifying significant causes of disease.

At the heart of this work is the idea that causes of disease can be identified long before we know the full biological story behind that disease. Austin Bradford Hill's famous guidelines for causal inference feature biological plausibility as one of nine desiderata, and urge caution in the application of this particular guideline (Hill 1965). Encouraged by the success with smoking and cancer, some epidemiologists went as far as to suggest that diseases should be treated as black boxes in epidemiological research. In particular, Richard Doll and Richard Peto argued in the early 1980s that many of the causes of cancer could already be identified, on the basis of statistical analysis of data that was either already available or else could be gathered without new technology or advances in the biomedical understanding of cancer etiology (Doll and Peto 1981).

\footnotetext{
${ }^{10}$ Alfredo Morabia reserves "modern" for the developments occurring from the $1980 \mathrm{~s}$ to the present day, and calls the period 1945-80 "classic" (Morabia 2004, 121-2). I am rolling both periods into the term "modern", because the important features for my argument are common to both.

${ }^{11}$ The most recent installment in this series of studies is (Doll et al. 2004).
} 
The "causes of cancer" project has been criticised on conceptual grounds, ${ }^{12}$ and its public health promise has not been fulfilled. Nonetheless, risk factor thinking is alive and well, indeed characteristic of modern analytic epidemiology (Greenland, GagoDominguez, and Casteleo 2004). Risk factors are "antecedents of adverse health outcomes that remain associated with the outcomes after adjustments for measured potential confounders" (Greenland, Gago-Dominguez, and Casteleo 2004, 529). Risk factor epidemiology involves looking for these associations, and (ideally) establishing whether they are causal. It is integral to this approach that an association need not be perfect in either direction in order to count as causal. For example, smoking is a risk factor for lung cancer: but not all smokers get lung cancer; and not all cases of lung cancer occur among smokers. Nevertheless, smoking is a causal risk factor for lung cancer: meaning the association is due to the fact that, in some individuals who smoke, smoking is a cause of lung cancer.

Risk factor thinking required a conceptual shift in the way that epidemiologists thought about disease. In the late nineteenth century, it was discovered that some diseases are caused by tiny organisms invading the body. This led to the idea - the hope - that a specific pathogenic organism could be discovered for every disease. As Robert Koch expressed the conviction:

...each disease is caused by one particular microbe - and by one alone. Only an anthrax microbe causes anthrax; only a typhoid microbe can cause typhoid fever. (Koch 1876; cited in Evans 1993, 20)

Subsequent empirical work showed that Koch's view of diseases as all caused by microbes was too restrictive. Diseases caused by deficiencies (e.g. scurvy), genetic disorders (e.g. Huntingdon's disease) and arguably even sub-cellular biological agents (e.g. viruses, prions) do not qualify; yet it seems arbitrary to deny that they are diseases on this basis alone. But there is also a conceptual aspect to Koch's view: that

\footnotetext{
12 The black box approach has been charged with a lack of explanatory specificity (Vandenbroucke 1988). An authoritative text book argues that Doll and Peto commit the error of supposing that attributable fractions could be summed, and should sum to $100 \%$, thereby overestimating the significance of their risk factors (Rothman, Greenland, and Lash 2008, 14-5). I have also discussed the black box approach more fully in another paper (Broadbent 2011).
} 
each disease has a defining cause, without which no set of symptoms can amount to a case of that disease.

Codell Carter makes a compelling case that this conceptual aspect is separable from the view that microbes are necessary for disease. He argues that necessary causes of disease are not discovered: rather, the disease is defined so as to make the cause in question essential to the disease. As he puts it:

If hydrophobia is an extreme inability to swallow, it really can be caused by blows to the throat, by psychological factors, or by the bites of rabid dogs... As long as diseases were defined in terms of symptoms, different episodes of any one disease simply did not share a common necessary cause. And no research, however brilliant, can find what isn't there. (Carter 2003, 37)

The view that diseases should be defined by reference to a single cause has been called the monocausal model of disease. ${ }^{13}$ It is not the view that diseases have only one cause, but rather that each disease should be defined by reference to one cause, which is necessary for the disease (by definition) and sufficient, in the right circumstances, to bring it about (Broadbent 2009, 303-5).

Risk factor thinking is not a good fit for the monocausal model. If, like Koch, you are committed to the view that one cause of a given kind of disease is of overriding salience, then cataloguing the various factors that predispose towards a disease without being either necessary or (circumstantially) sufficient is of dubious benefit. ${ }^{14}$ Jacob Henle, a teacher of Koch's, puts the point wonderfully (in a passage cited by Carter):

Only in medicine are there causes that have hundreds of consequences or that can, on arbitrary occasions, remain entirely without effect. Only in medicine

\footnotetext{
${ }^{13}$ Carter calls it the etiological standpoint (Carter 2003). However my own previous formulation (monocausal model) is preferred here as more precise. The view implies a numerical restriction on the number of defining causes to one (for reasons given in Broadbent 2009, 303). Incorporating necessity and sufficiency into a multi-causal model is a non-trivial project.

${ }^{14}$ Except insofar as it helps identify a cause meeting those criteria.
} 
can the same effect flow from the most varied possible sources. One need only glance at the chapters on etiology in handbooks or monographs. For almost every disease, after a specific cause or the admission that such a cause is not yet known, one finds the same horde of harmful influences - poor housing and clothing, liquor and sex, hunger and anxiety. This is just as scientific as if a physicist were to teach that bodies fall because boards or beams are removed, because ropes or cables break, or because of openings, and so forth. (Henle 1844; cited by Carter 2003, 24)

The kinds of event Henle lists are all risk factors for falling, and moreover causal ones. He is not denying that ropes breaking, boards being removed, and the rest are causes of objects falling; he is denying that they are all equally relevant for scientific purposes.

Modern epidemiology, on the other hand, does regard collecting information about risk factors for disease as a scientifically fruitful activity. It is therefore unsurprising that modern epidemiology should have endorsed a more permissive model of disease, one which does not differentiate so radically between those causes of disease which a self-respecting scientist may cite in explanation and those which she may not.

On the multifactorial model, a given kind of disease arises from a number of different factors, and there is no requirement that one of these factors be present in every case of the disease, nor sufficient for the disease in any circumstances (at least any that are specifiable). Perhaps the best-known expression of this idea is MacMahon and Pugh's "causal web" metaphor (MacMahon and Pugh 1970). The idea is that each disease sits in a "web" of factors, causally interacting with each other as well as with the disease outcome in question. The metaphor is generally applied at the level of types rather than tokens, but in principle there is no reason why it should not be applied to individual cases of disease rather than to generalisations about disease etiology.

Neither the causal web model, nor multifactorialism more generally, have the resources to give any sense to the idea that some risk factors are more salient or more important for some purposes than others. We might distinguish between those factors that are more or less "proximate", as did some nineteenth century medical texts; but 
this approach is notoriously hard to make precise. We might rank the importance of causes by the strength of the association they explain, measured on some standard epidemiological measure. But which one? There are a number of measures, and what looks like a strong association on one measure might look weak on another. For example, the number of excess deaths due to heart disease in smokers is much higher than the number of excess deaths due to lung cancer, and heart disease is the leading smoking-related cause of death (Morabia 2004, 63). But the relative risk is much higher for lung cancer (in the order of 10) than coronary heart disease (in the order of <2) (Cornfield et al. 1959; Poole 2010). This is a complex issue, and a topic of ongoing debate in the epidemiological literature (see especially Poole 2010 and generally the January 2010 issue of Epidemiology). It is highly doubtful, therefore, that any particular measure can be identified with causal "salience".

One illustration of this shortcoming of the multifactorial model is in its handling of infectious diseases. Take, for example, tuberculosis. Notwithstanding the shortcomings of the monocausal model, there is some sense in attempting to single out infection with tubercle bacilli among the various causes of tuberculosis. For many purposes, this is a particularly salient cause: for the purposes of immunisation, treatment, quarantine, prognosis, and so on. But if we draw a causal web for tuberculosis, it will not have any particular structural features that indicate this salience. In some populations, the web may feature many of the same items that feature on a web for many quite different diseases, such as obesity. And even if it does not share the same items, the structure of the web will not be notably different. Bacilli infection will take a place alongside nutrition, and below socioeconomic status, for example.

Adopting the multifactorial model does not prohibit epidemiologists from acknowledging the salience that bacilli infection has for many practical and scientific purposes. But nor does it encourage them to do so. Nor does it provide them with a means to express the importance of this cause for particular purposes. And, in further consequence, it does not provide any opportunity to specify what those purposes might be. 
There is considerably more that might be said both for and against the multifactorial model of disease in general. But our concern is with neglected disease. In the next section I shall explain how this shortcoming of the multifactorial model makes it illsuited to defining neglected diseases, for both scientific and political purposes.

\section{Multifactorialism and neglected diseases}

To define neglected disease, we must define disease. Multifactorialism, as it has developed in modern epidemiology, is useless for this task. It says nothing at all that is distinctive about disease. Everything in the natural world is multifactorial, with the possible exception of the Big Bang. It is possible to draw a causal web for the building of houses, the collapse of economies, the birth of children, the explosion of supernovae. ${ }^{15}$ So saying that $X$ is multifactorial does not place much of a restriction on what $X$ could be. To say that a disease is multifactorial is to say very little about it at all: hence the need, in the previous section, to explain multifactorialism in contrast to the more restrictive monocausal model.

Not only is multifactorialism useless for defining disease, it is also useless for the related task of defining diseases. It does not tell us what makes $X$ one disease rather than another, any more than it tells us what makes $X$ a disease in the first place. On Carter's compelling analysis, the monocausal model was in large part a thesis about how diseases should be defined - that is, distinguished from one another. The proposal was that they should be defined by picking a cause, and defining the disease with reference to that cause. Reference to symptoms must necessarily be made, since the cause must be a cause of something; but the same symptoms without the defining cause do not amount to a case of the disease in question. The multifactorial model, as we have seen, removes this restriction on disease definition. But it does not propose any replacement.

\footnotetext{
${ }^{15}$ The same is true if the web is applied to the etiology of particular events rather than types of event.
} 
These difficulties are quite general, and not specific to neglected diseases. ${ }^{16}$ My main concern in this section is to argue that the multifactorial model of disease is potentially damaging specifically for thinking about, and trying to do something about, neglected diseases. If this were not so, then we could happily regard the task of defining disease as a purely philosophical past-time, and satisfy ourselves for practical purposes with a definition of neglect alone. But I think that the multifactorial approach to disease, which pervades modern epidemiology, has the potential to disrupt and divert efforts to reduce the burden of neglected diseases on global population health. For this reason, it is worthwhile to include a definition of disease in our definition of neglected disease.

There are two related ways in which multifactorial thinking might be detrimental to thinking and doing something about neglected diseases. First, it does not provide a ready distinction between those diseases that are well-understood and those that are not. Gathering risk factors appears as the primary epidemiological research activity, but in the case of many neglected diseases, it is next to useless: because the disease is sufficiently well understood to devise effective public health interventions. Let us take yaws as an example again. If the purpose is identifying means of preventing or curing yaws, then gathering information on the risk factors for yaws is of limited use. We have a cure, and we know how the disease is transmitted (skin-to-skin contact). Of course, the multifactorial model does not prevent us from acknowledging the fact that we already understand yaws relatively well, for medical and public health purposes. But nor does it give us any ready means to express this fact.

Even if the purpose of our research is not practical, but purely scientific, it is not clear how useful it is to know more about the risk factors for yaws. From a biological perspective, many of these may be irrelevant, because contingent. For example, socioeconomic status and geographical location are causal risk factors for yaws. But they are not part of the domain of biology. They are, from the biological perspective, contingent facts about how the various components of a case of yaws happen to come together. They are not part of the biological story about yaws. It might be replied that

\footnotetext{
${ }^{16}$ For detailed criticisms of the multifactorial model in general, including an argument that it represents an uncritical reversion to older ways of thinking about disease, see (Broadbent 2009).
} 
the biological perspective is not the same as the epidemiological perspective, and that a full scientific understanding of disease requires considering "social causes" of disease. Thus, for example, Nancy Krieger has argued that attention to socioeconomic factors is necessary for a full scientific understanding of disease (Krieger 2007). But even if this is correct (and I am not taking a stance on that issue here), it is hard to see how it motivates further research into socioeconomic risk factors in the case of yaws. We already know that yaws afflicts very poor people in certain tropical locations. Krieger does not have any argument that more research into socioeconomic risk factors is always necessary, regardless of the current state of knowledge. My point applies to research into social determinants as much as pharmacological research: in the case of yaws, neither represents the most effective strategy for reducing either the incidence or the prevalence of the disease. The reason is the same, namely, that the disease is well understood, and means for controlling it already exist.

This point is a consequence of the one I pressed at the end of the previous section, namely, that the multifactorial model has no way of marking out what is special about (e.g.) the tubercle bacilli, among all the other causes of tuberculosis. Because it cannot do this, it cannot mark the difference between a situation where we have identified a cause that is, for our purposes, particularly important, and the situation where we have not done so. In the former situation, it is far less likely that cataloguing risk factors is a useful activity.

My second point about the implications of multifactorialism for neglected disease is a practical corollary of the first, namely, that the multifactorial model encourages a distorted view of the correct practical response to neglected diseases. The multifactorial model provides no way to distinguish between worthwhile investment in R\&D, and investment that is not worthwhile. In Section 2 I argued that this is an important distinction to make, and I criticised some extant proposals for reducing the burden of neglected diseases, on the basis that they wrongly emphasize R\&D investment. The multifactorial model of disease, and the associated conception of the goal of epidemiological research as gathering knowledge about risk factors, lend themselves to this incorrect emphasis. 
I do not mean to suggest that R\&D investment in diseases that are understood including some neglected diseases - automatically fails to be worthwhile. There may be diseases which are relatively well understood, but for which more research is necessary. The simplest such case is where we lack effective cures - for example, there is a need for development of drugs to combat extensively drug-resistant (XDR) tuberculosis. ${ }^{17}$ Diagnosis provides another example: developing a reliable diagnostic test may remain an elusive research goal even after a disease is relatively wellunderstood, for example if it is asymptomatic in early stages, or symptomatically resembles another disease.

The point, rather, is that merely finding things out is not necessarily useful, for either scientific or practical purposes. The multifactorial model, and risk factor thinking, are not equipped to mark a distinction between useful and non-useful directions of research.

My contention might sound far-fetched. Can a model of disease really matter that much? Can it influence research and shape public health policy? Moreover, has it in fact done so in the case of neglected diseases?

I would answer all these questions in the affirmative. Carter makes a strong case that the conceptual shift towards etiological classification of disease played a part in some of the medical success stories of the early twentieth century - notably the development of antibiotics and vaccination (Carter 2003). Defining diseases by their causes makes developing and deploying these tools considerable more straightforward. Conceptual developments in our thinking about disease can be practically important; this is a feature of the general point that concepts can be more or less useful for given purposes. What is more, these purposes may include financial interests. For example, Koch's insistence on specificity of microorganism for disease was aligned not only with the public interest in reducing the incidence of certain diseases, but also with his own interest in selling vaccines. I am not making any claim about Koch's motivation; the point is that financial interests, like any other purposes,

\footnotetext{
${ }^{17}$ Tuberculosis is not generally counted as a neglected disease: I am merely using it to illustrate the point that further R\&D can be useful even when curative measures already exist.
} 
can be better or worse served by - and therefore, presumably, can shape - concepts of disease.

There is some evidence that the multifactorial model of disease has on occasion misdirected $R \& D$, and moreover that it has served financial interests in doing so. The example I have in mind is the well-known discovery of the role of the bacterium Helicobacter pylori in the etiology of peptic ulcer. Previously a number of risk factors had been identified. Ulcer was thought to be caused by an excess of hydrochloric acid in the stomach, brought on by stress, spicy food, or other unknown factors. Pharmaceutical interventions had been developed to control the acidity in the stomach. But in the early 1980s, Robin Warren and Barry Marshall identified a bacterium, Helicobacter pylori, as causally associated with gastric ulcer (Marshall and Warren 1984a; Marshall and Warren 1984b). The claim was poorly received, so Marshall famously gathered further evidence by self-experimentation. He drank a culture of the bacteria and developed ulcer, then took a short course of antibiotics to rid himself of it (Marshall, Armstrong, et al. 1985; Marshall, McGechie, et al. 1985).

The initial resistance to this discovery is hard to explain except by reference to the multifactorial model of disease. If Koch's dicta had still been dominant, then the claim that stomach ulcer was caused by an organism, and that killing the organism would remove the disease, ought not to have come as a surprise. The claim that the organism had been identified might still have been treated with scepticism; but the form of the claim - that some single organism was responsible for peptic ulcer would have seemed unexceptional. But it seems that this was precisely what generated the scepticism. This is hard to explain except by reference to the fact that the multifactorial model exerts no pressure to find a cause with any special properties, certainly not one which is present in all cases of disease and sufficient, in the right circumstances, to bring it about.

It is often remarked that $H$ pylori does not satisfy these conditions. It does not account for all stomach ulcer, and many people have $H$ pylori without developing ulcer. But this is no different to the situation that faced late nineteenth century physicians who identified organisms that caused diseases such as cholera. Vibrio cholerae accounts for only a fraction of all diorrhea. Tuberculosis bacilli can lie dormant in the lungs. 
The former kind of exception may be dealt with by definition, as discussed in the previous section; the latter, by further research into what makes the difference between asymptomatic and symptomatic infections.

Moreover, and interestingly, the discovery of $H$ pylori was not profitable. Long term symptomatic treatments are a good deal more lucrative than a short course of antibiotics. I am not asserting that financial interests played any role in the slow initial response to H pylori's discovery: I have no non-circumstantial evidence either way. Rather, I am emphasizing the point made in Section 2, that the most profitable means to reduce the disease burden are not always the most effective means.

It is arguable that a similar distortion of $R \& D$ into diabetes currently exists. Type 1 diabetes is the inability to produce insulin, arising because the immune system attacks and kills the pancreas at some point in childhood. In recent years, the management of diabetes has been improved by the development of new pharmacological insulin treatments, which make it easier for a diabetic to maintain a more constant blood sugar level. Developing slow-acting insulin is of course extremely useful, but it does not get us any closer to understanding why diabetes occurs in the first place.

Moreover the financial incentives for developing a symptomatic treatment for a lifelong condition are very great. ${ }^{18}$

Diabetes attracts considerable R\&D funding per DALY. ${ }^{19}$ Even so, it is possible at least to ask whether biomedical science is well-ordered in respect of diabetes. This should give pause to those who emphasize $R \& D$ as the primary strategy to reduce the burden of neglected diseases.

To summarize: the multifactorial approach to disease that has developed in modern epidemiology is ill suited to the project of defining neglected disease.

Multifactorialism, in its current form, says nothing distinctive about disease, and is for this reason quite useless for the general philosophical project of identifying at least some criteria that phenomena must satisfy in order to count as diseases. Moreover, multifactorialism is a particularly poor fit for neglected diseases. (This should come

${ }^{18}$ This point is discussed in (Angel 2008).

${ }^{19} \$ 102.07$, according to Reiss and Kitcher $(2009,264)$. 
as no surprise, since modern epidemiology has developed in countries which have passed through the epidemiological transition.) This way of thinking about diseases encourages the view that cataloguing risk factors is a worthwhile activity; yet for many neglected activities, it is of limited use, either for scientific or public health purposes. Moreover, it does not provide any tools for distinguishing between research that is worthwhile and research that is not. This encourages the mistaken emphasis on $R \& D$ funding as a solution for neglected diseases, at the expense of more pressing concerns. As MacMahon and Pugh pointed out when they introduced the causal web metaphor, most of the web will remain unknown to us. Moreover, even where R\&D is a good strategy, it is important to distinguish good from bad directions of research. We saw two examples of R\&D into non-neglected diseases whose well-orderedness was questionable, despite reducing the burden of these diseases (by improving management). The trouble is that reducing the burden is not necessarily the same thing as reducing the burden profitably, as the discovery of $H$ pylori demonstrates. In the story of $H$ pylori's discovery, and possibly even in the case of type 1 diabetes, the multifactorial approach prevalent in modern epidemiology has not proved helpful.

What the multifactorial model does provide, however, is ample opportunities for research. The multifactorial model provides no clear direction for this research; there is no inbuilt way to judge whether it is fit for purpose, since there is no way of plugging a purpose into the multifactorial model. This means that the multifactorial model is well-suited to R\&D whose purpose, if clearly identified, might not appear very attractive: for example, research that is designed to identify opportunities for profit. The model makes it very hard to criticise investment as long as the results are well supported by evidence, even if the claims that are well supported are unimpressive. For example, a great deal of money is spent on developing pharmaceutical interventions for cardiovascular diseases. These interventions are designed to operate on various risk factors for cardiovascular disease, such as blood pressure and cholesterol. These investments are out of all proportion to their impact on population health. The multifactorial model is complicit in obscuring that fact, because (as we saw in the previous section) it does not provide any ready way to distinguish between the relative importance of risk factors for any purpose. It does not prohibit distinguishing good from bad research; but nor does it lend itself to making that distinction. Similarly, vaccines could have been administered within an older, 
symptomatic view of disease definition; but the fit would not have been good. There was a strong case for conceptual retooling. I suggest there is a similar need for conceptual retooling in contemporary discussion of neglected diseases. In the next section I will endorse a model of disease that is better suited to defining neglected diseases, and doing something about them.

\section{$\underline{\text { 5. The contrastive model }}$}

My suggestion is that the contrastive model of disease that I have previously proposed (Broadbent 2009, 308) is apt for neglected diseases. The purpose of that model is to retain the methodological advantages of the monocausal model, but without any restriction in the number of defining causes that a disease may have. To that extent the model might be seen as a development of the multifactorial approach that currently dominates modern epidemiology - a more sophisticated kind of multifactorialism. Here is the model:

For person $p$ to have disease $D$, it is necessary that:

SYMPTOMS $p$ suffers from some of a set of symptoms of ill health $S$, which are differences between $p$ and a contrast class $X$;

CAUSES Among the causes of $p$ 's symptoms are events of kinds $C_{l}, \ldots$ $C_{n}$, at least some of which are not causes of the absence of the symptoms $S$ from each member of $X$. (Broadbent 2009, p. 308)

Put more plainly, the two requirements of this model are as follows. First, a disease must have a set of typical symptoms, at least some of which must be present at some point in time for a case of the disease to exist. Long latency periods are therefore allowed, but entirely asymptomatic diseases are not. Since the neglected diseases have symptoms, and indeed are problematic in virtue of their symptoms, this is not a problematic requirement in the present context. The symptoms are defined with 
reference to a contrast class, ${ }^{20}$ who do not suffer the symptoms. Note that I am ignoring the distinction drawn in medical contexts between a symptom and a sign, where a symptom is reported by the patient, and a sign is discovered by medical examination. I am using "symptom" in a non-technical sense to cover both of these things; thus a headache and a swollen appendix could both satisfy SYMPTOMS. ${ }^{21}$

Second, the model requires that the symptoms be caused in a particular way. More exactly, there must be one or more causes which are present together in every case of the disease, but at least one of which is absent from some contrast class (or more precisely, does not cause the absence of symptoms from that contrast class). ${ }^{22}$

In a previous paper I have defended this model of disease in general, but here I want to suggest that it is apt for neglected diseases in particular, and to add a simple further criterion for a disease to count as neglected. Its aptitude arises from the fact that it poses constraints on what can count as a disease, constraints which are severe and yet which neglected diseases typically satisfy. Yaws, for example, satisfies SYMPTOMS, because a set of typical symptoms (spread over a certain time period) can be described that mark differences from healthy people. And it satisfies CAUSES in virtue of the bacterium which - it is so natural to say - causes the disease. This bacterium is never a cause of the lack of symptoms - it is never a cause of any other characteristic set of symptoms, or of good health. Similar remarks apply to infectious and parasitic diseases quite generally, and the neglected diseases are largely infectious or parasitic.

We are now in a position to say that a neglected disease is one which satisfies SYMPTOMS, CAUSES and NEGLECT. This amounts to a tripartite definition of neglected disease.

\footnotetext{
${ }^{20}$ The contrast class may be partly or wholly counterfactual.

${ }^{21}$ I am grateful to an anonymous referee for alerting me to the distinction between these terms in medical contexts.

${ }^{22}$ There is a great deal more to be said both in defence of and objection to this model, which I omit here in favour of a focus on its application to neglected diseases. In particular, the model requires an independent definition of health to fix its contrast classes. This puts the model at odd with theories of health such as Christopher Boorse's, which seek to define health as the absence of disease. See (Boorse 1975; Kingma 2007)
} 
There are two obvious questions to ask of this definition. First, can it really be right to exclude from any list of neglected diseases a health condition satisfying NEGLECT but failing the contrastive definition of disease? Second, how does adopting this definition help with the problems previously identified with the unrestricted multifactorial approach?

Regarding the first question, the best place to start with is with a list of neglected diseases, for example, that provided by the WHO. Those diseases are mostly infectious or parasitic, and for that reason they are a good fit for etiological approaches to disease definition. The exception is snakebite, which is neither infectious nor parasitic. Moreover, different snakes have different venom, producing different symptoms, and requiring different treatments. In a clinical context, we would be unlikely to use the classification "snakebite" to indicate a single condition, for these reasons. However, the advantage of an explicitly contrastive model is its sensitivity to the contrasts we have in mind. In the clinical context, these contrasts may be more fine-grained than in the public health context. It seems to be so in the case of snakebite. For public health purposes, there may be reasons to treat snakebite as a single disease, in particular arising from the public health measures required. If there are such reasons (I shall indicate some in the next paragraph), then the contrastive model can count snakebite as a disease for public health purposes. The WHO's more exact definition is "envenoming resulting from snake bites" (World Health Organisation 2010d), which picks out a common cause of all cases, satisfying CAUSES. It is easy to make SYMPTOMS sufficiently broad, either by cataloguing the effects of various kinds of snake venom, or by sticking with SYMPTOMS itself, so that any kind of ill-health caused by snake venom is included.

Snakebite provides a good example of the utility of classifying diseases according to their etiology. For public health purposes, this classification is useful. For clinical purposes, and also for the purposes of $R \& D$, it might be less useful, since at the biological and chemical level, envenomation due to snakebite is etiologically diverse, because venoms differ. This contrast provides another excellent illustration of the dangers of thinking of neglected diseases in terms of R\&D investment. Despite its biochemical diversity, it is sensible to treat snakebite as one disease for public health 
purposes, because the interventions that are required to reduce its burden are not at the biochemical level. According to the WHO, "poor access to health services... and, in some cases, a scarcity of antivenom, often leads to poor outcomes and considerable morbidity and mortality" (World Health Organisation 2010d). It is very hard to see how R\&D investment can improve access to health services in poor rural areas. It might encourage the manufacture of antivenom, by offering new opportunities for profit. But any such opportunity would need to be subsidised by some sort of incentive scheme such as Pogge's, given the inability of most snakebite sufferers to pay. And it is hard to see how incentivising the development of a new anti-venom could be cheaper than incentivising the manufacture of an existing one.

It seems, then, that all the diseases on the WHO's list of neglected diseases fit the contrastive model of disease, including the non-infectious item on that list. Moreover the contrastive model provides justification for treating snakebite as a single disease for public health purposes, even if it is not a single disease for clinical purposes. The question as to whether a disease would be excluded from a neglected disease list for failing the contrastive model remains hypothetical. But I think the answer is that it probably would be excluded, and should. The point about neglect is that there is a course of action that could be taken - including, but not limited to, more R\&D investment - but which is not taken. It is far less likely that any such course of action will exist for health conditions failing to satisfy the contrastive model of disease.

In effect, insisting on strict criteria for neglected diseases serves to prevent dilution of the pool of neglected diseases with conditions which, while they may pose major public health problems, are less readily actionable, and thus less dramatically unjust. To take an example: obesity in the developed world is closely negatively correlated with income. Some have argued that correlations of this sort are wrongly neglected by epidemiologists (e.g. Krieger 2007). Suppose, for the sake of argument, that obesity satisfies NEGLECT in this respect (notwithstanding the substantial research efforts concerning the genetics of obesity ${ }^{23}$ ). Even on this supposition, obesity ought not thereby to qualify as a neglected disease, if the current significance of that phrase is to be retained. Obesity (where not the result of certain very rare monogenetic disorders)

${ }^{23}$ E.g. (Farooqi and O'Rahilly 2006). For a recent estimate of the relative contribution of genes and environment, see (Wardle et al. 2008). 
is typically mediated by what we would normally recognise as choices made by the sufferer. Social injustice may be part of the story, but if so, the story is complex and the correct remedy is unclear. For this reason, we should not count obesity as a neglected disease, even if it is sometimes or in some ways neglected.

Contrast obesity with hookworm, which arises from hookworm larvae penetrating the skin and making their way to the small intestine. Genetics are largely irrelevant; and social problems are relevant only insofar as they differentially expose people to hookworm larvae. Poverty causes ill health the world over, but it is not in the neglected disease activist's interest to hitch her wagon to the much more complex project of identifying social determinants of health. ${ }^{24}$ The distribution of obesity may be unjust, in some sense; but the distribution of hookworm is considerably more unjust. And the reason for this has to do with the kinds of diseases that they are. Hookworm is well understood, preventable by provision of basic sanitation, and readily treatable. Obesity, of the kind that is most prevalent, is less well understood: it is a complex social and psychological problem, possibly with some genetic components; and the public health measures required to reduce its burden are far from clear.

It seems, then, that there are good grounds for excluding health conditions that fail the contrastive model of disease from any list of neglected diseases. Part of the injustice attendant on neglected diseases stems from the kind of disease they are. They are not merely health-related woes of a general kind. They are specific diseases with specific causes, and specific steps could be taken to prevent, cure or manage them, or to improve the availability of preventive, curative or mitigatory measures. The contrastive model of disease brings out this feature.

The second question we might ask of the proposed tripartite definition of neglected disease is how adopting the contrastive model helps with the problems identified in the previous section for the unrestricted multifactorial approach. The short answer is that it does not suffer from the shortcomings of that approach. The contrastive model does discriminate among causal risk factors. This does not imply that all attention

${ }^{24}$ A good statement of that project is (Marmot 2006). An excellent survey of the various possible causal connections between health and income is (Deaton 2003). 
must be focused on the causes satisfying CAUSES. But any proposed course of action must ultimately be justified in relation to those causes, however removed the action may be, since it is only by somehow preventing those causes from having their customary effects ${ }^{25}$ that the public health burden of the disease will be reduced. Steps to reduce the number of mosquitoes in areas where dengue fever is prevalent are justified by the fact that the mosquitoes are a vector for the virus. Likewise, R\&D for a vaccine is justified to the extent that the vaccine might offer some resistance to the virus. The less impact a measure has (for a given expenditure) on the cause in CAUSES, the less justified it is. If a vaccine would be too expensive, or the infrastructure too poor to implement a vaccination program, then the relative merits of measures to reduce the mosquito population become greater.

Adopting the proposed definition of neglected diseases would thus at least avoid the problems that I have attributed to the dominance of multifactorial thinking. More positively, it would make it easier to assess the reasonableness of proposed strategies for handling neglected diseases. For example, it enables us to say that Pogge's suggestion that R\&D investment be rewarded in proportion to its impact on global population health is too narrow. In many cases, the largest impact on global population health is to be had by doing something other than investing in R\&D. Unrestricted multifactorialism - typified by the causal web metaphor - has no resources to express this fact. It does not prevent us pointing it out; but nor does it help us. The contrastive model, on the other hand, can express it clearly. In many cases, $R \& D$ is not the most effective way to inhibit or prevent the causes of the disease or prevent them from occurring - where "the causes" means those which satisfy CAUSES.

\section{Conclusion}

Neglect is not best characterised purely in terms of R\&D, but in terms of the differential availability of preventive, curative or mitigatory measures, or of efforts to obtain those measures. The trend towards unrestricted multifactorialism in modern epidemiology encourages a mistaken emphasis on $R \& D$ in strategies to reduce the

\footnotetext{
${ }^{25}$ Whether by preventing them from occurring or by inhibiting their action.
} 
burden of neglected diseases on global population health. It should be corrected by adopting a more restrictive model of disease such as the contrastive model endorsed here. It may seem surprising, prima facie, that a philosophical project like defining disease should have practical consequences. But concepts can be more or less fit for purpose, including practical purposes, and there is no reason to suppose that the concept of disease is an exception. There is, moreover, some empirical basis for doubts about unrestricted multifactorialism. Accordingly I suggest that neglected diseases be defined as satisfying NEGLECT, SYMPTOMS and CAUSES, as those have been defined here. ${ }^{26}$

\section{$\underline{\text { References }}$}

Angel, Katherine. 2008. Causality and Psychosomatic Histories in Contemporary Anglo-American Biomedicine. PhD, University of Cambridge.

Boorse, Christopher. 1975. On the distinction between disease and illness. Philosophy of Public Affairs 5: 49-68.

Broadbent, Alex. 2009. Causation and models of disease in epidemiology. Studies in History and Philosophy of Biological and Biomedical Sciences 40: 302-311. . 2011. Causal inference in epidemiology: mechanisms, black boxes, and contrasts. In Causality in the Sciences, ed. Phyllis McKay Illari, Federica Russo, and Jon Williamson, 45-69. Oxford: Oxford University Press.

Burri, Christian. 2004. Research on Neglected Diseases. Bulletin of Medicus Mundi Switzerland 92.

http://www.medicusmundi.ch/mms/services/bulletin/bulletin200401/kap01/08 burri.html.

Carter, K. Codell. 2003. The Rise of Causal Concepts of Disease. Aldershot: Ashgate.

Cornfield, Jerome, William Haenszel, E. Cuyler Hammond, Abraham M. Lilienfeld, Michael B. Shimkin, and Ernst L. Wynder. 1959. Smoking and lung cancer: recent evidence and a discussion of some questions. J. Nat. Cancer Inst. 22: 173-203.

Deaton, Angus. 2003. Health, Inequality, and Economic Development. Journal of Economic Literature 41, no. 1: 113-158.

DiMasi, J., R. Hansen, and H. Grabowski. 2003. The Price of Innovation: New Estimates of Drug Development Costs. Journal of Health Economics 22: 151185.

Doll, Richard, and Richard Peto. 1981. The causes of cancer. Oxford: Oxford University Press.

Doll, Richard, Richard Peto, Jillian Boreham, and Isabelle Sutherland. 2004.

${ }^{26}$ I am grateful to Dan Brock, Alena Buyx, the audience at the Brocher FoundationLSE Workshop on Neglected Diseases in December 2008, and especially to the anonymous reviewers for their many helpful comments and corrections. I am also grateful to the PHG Foundation and the Brocher Foundation for supporting this work. 
Mortality in relation to smoking: 50 years' observations on male British doctors. British Medical Journal 328, no. 7455 (June 26): 1519. doi:10.1136/bmj.38142.554479.AE.

Evans, Alfred S. 1993. Causation and Disease: A Chronological Journey. New York, N.Y.: Plenum Publishing Corporation.

Farooqi, Sadaf, and Stephen O'Rahilly. 2006. Genetics of Obesity in Humans. Endocrine Reviews 27, no. 7: 710-718.

Fegan, David, Mary J Glennon, Yogendra Thami, and George Pakoa. 2010. Resurgence of yaws in Tanna, Vanuatu: time for a new approach? Tropical Doctor 40, no. 2 (April 1): 68-69. doi:10.1258/td.2009.090249.

Framingham Heart Study. 2010. Framingham Heart Study. September 10. http://www.framinghamheartstudy.org/.

Greenland, Sander, Manuela Gago-Dominguez, and Jose Esteban Casteleo. 2004. The Value of Risk-Factor ("Black-Box") Epidemiology. Epidemiology 15, no. 5: 529-535.

Henle, Jacob. 1844. Medicinische Wissenschaft und Empirie. Zeitschrift fur rationelle Medizin 1: 1-35.

Hill, Austin Bradford. 1965. The environment and disease: association or causation? Proceedings of the Royal Society of Medicine 58: 259-300.

Kingma, Elselijn. 2007. What is it to be healthy? Analysis 67, no. 2: 128-133.

Kitcher, Philip. 2001. Science, Truth and Democracy. Oxford: OUP.

Koch, R. 1876. Verfrahen sur Untersuchung zur conserviren und photographie der Bakterien, Beitrag der Pflanzen. Breslow: Cohn's Bier.

Krieger, Nancy. 2007. Why Epidemiologists Cannot Afford to Ignore Poverty. Epidemiology 18, no. 6: 658-663.

MacMahon, B., and T.F. Pugh. 1970. Epidemiology: Principles and Methods. Boston: Little, Brown.

Marmot, Michael. 2006. Health in an unequal world: social circumstances, biology, and disease. Clinical Medicine 6, no. 6: 559-572.

Marshall, B.J., J.A. Armstrong, D.B. McGechie, and R.J. Glancy. 1985. Attempt to fulfil Koch's postulates for pyloric Campylobacter. Medical Journal of Australia 142: 436-439.

Marshall, B.J., D.B. McGechie, P.A. Rogers, and R.J. Glancy. 1985. Pyloric Campylobacter infection and gastroduodenal disease. Medical Journal of Australia 142: 439-444.

Marshall, B.J., and J.R. Warren. 1984a. Unidentified curved bacilli in the stomach patients with gastritis and peptic ulceration. Lancet 1, no. 8390: 1311-5. doi:10.1016/S0140-6736(84)91816-6. . 1984b. Unidentified curved bacilli in the stomach patients with gastritis and peptic ulceration. Lancet 1, no. 8390: 1311-5. doi:10.1016/S01406736(84)91816-6.

Morabia, Alfredo. 2004. History of epidemiologic methods and concepts. Basel: Birkhauser Verlag.

Pogge, Thomas. 2005. Human Rights and Global Health: A Research Program. Metaphilosophy 36: 182-209.

Poole, Charles. 2010. On the Origin of Risk Relativism. Epidemiology 21, no. 1 (1): 3-9. doi:10.1097/EDE.0b013e3181c30eba.

Reiss, Julian, and Philip Kitcher. 2009. Biomedical Research, Neglected Diseases, and Well-Ordered Science. Theoria 66: 263-282.

Rothman, Kenneth J, Sander Greenland, and Timothy L Lash. 2008. Modern 
Epidemiology. 3rd ed. Philadelphia: Lippincott Williams \& Wilkins.

Saracci, Rodolfo. 2010. Epidemiology: A Very Short Introduction. Oxford: Oxford University Press.

The Carter Center Guinea Worm Disease Eradication Program. 2010. Treatment. September 2.

http://www.cartercenter.org/health/guinea_worm/index.html\#treatment.

Trouiller, Patrice, Pierro Olliaro, Els Torreele, James Orbinski, Richard Laing, and Nathan Ford. 2002. Drug development for neglected diseases: a deficient market and a public health policy failure. The Lancet 359, no. 9324: 21882194.

Vandenbroucke, J.P. 1988. Is 'The Causes of Cancer' a Miasma Theory for the End of the Twentieth Century? International Journal of Epidemiology 17, no. 4: 708709.

Wardle, Jane, Susan Carnell, Claire M.A. Howarth, and Robert Plomin. 2008.

Evidence for a strong genetic influence on childhood adiposity despite the force of the obesogenic environment. American Journal of Clinical Nutrition 87: 398-404.

World Health Organisation. 2010a. WHO | Diseases covered by NTD Department. October 4. http://www.who.int/neglected_diseases/diseases/en/. http://www.who.int/csr/disease/dengue/en/index.html. . 2010c. WHO | Dengue/dengue haemorrhagic fever. September 2. . 2010b. WHO | Control of Neglected Tropical Diseases. October 4. http://www.who.int/neglected_diseases/en/. - 2010d. WHO | Snakebite. September 10. http://www.who.int/neglected_diseases/diseases/snakebites/en/index.html. 